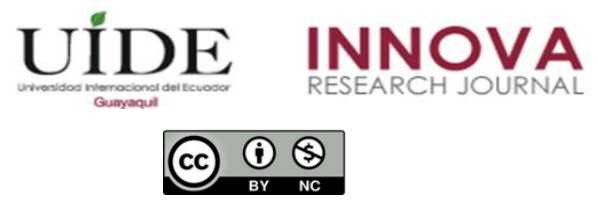

INNOVA Research Journal, ISSN 2477-9024

(Septiembre-Diciembre 2020). Vol. 5, No.3.1 pp. 300-314

DOI: https://doi.org/10.33890/innova.v5.n3.1.2020.1512

URL: http://revistas.uide.edu.ec/index.php/innova/index

Correo: innova@uide.edu.ec

\title{
Efecto de la luz artificial en la anidación de tortugas marinas en playas del Cantón Puerto López, Manabí, Ecuador
}

\section{Artificial light effects on the sea turtles nesting on the beaches of Puerto Lopez canton, Manabi Ecuador}

\author{
Renato Enrique Arízaga Medina \\ (D) https://orcid.org/0000-0003-4669-2631 \\ Luis Eduardo Cárdenas Pasato \\ Universidad Internacional del Ecuador, Ecuador
}

Autor para correspondencia: renatoarizaga@gmail.com; lucardenaspa@uide.edu.ec

Fecha de recepción: 24 de agosto de 2020 - Fecha de aceptación: 21 de octubre de 2020

\section{Resumen}

En muchas playas del mundo las actividades antropogénicas coexisten con los procesos naturales de las especies silvestres. En Ecuador las tortugas marinas no son la excepción, especies como: Laúd (Dermochelys coriacea), Boba (Caretta caretta) y Golfina (Lepidochelys olivacea) se ubican en la categoría de Vulnerables según la UICN; la Tortuga Verde (Chelonia mydas) En Peligro; las Tortuga Carey (Eretmochelys imbricata) y Bastarda (Lepidochelys kempii) En Peligro de Extinción. Debido a la vulnerabilidad de estos organismos, como resultado de las actividades humanas, el presente estudio evalúa uno de los factores que inciden en los procesos de anidación de estas tortugas, la incidencia de la luz artificial en cuatro playas del Cantón Puerto López, Manabí, como un elemento que impacta en la anidación y la alteración de los desplazamientos de los neonatos hacia el océano. Los resultados alcanzados en este estudio muestran que distintas zonas de paya tienen barreras contra la luz, siendo éstas de origen antrópico o natural favoreciendo a las distintas especies de tortugas que puedan anidar en estos lugares.

Palabras claves: tortugas marinas; anidación; conservación; sostenibilidad; luz artificial.

\begin{abstract}
On several beaches in the world, anthropogenic activities coexist with the natural processes of the wild species. In Ecuador, sea turtles are no exception; the leatherback turtles (Dermochelys coriacea), Boba (Caretta caretta) and Golfina (Lepdochelys olivacea) are in the vulnerable category; Green Turtle (Chelonia mydas) in danger; Hawksbill Turtle (Eretmochelys imbricata) and Bastard (Lepidochelys kempii) in danger of extinction. Due to this vulnerability resulting from human activities, the present study evaluates the incidence of artificial light on four beaches in the Puerto López canton, as an element that impact on the nesting and the alteration of the migration of the newborns to the ocean. The results reached in this study show with different areas of the clown has barriers against light being this of anthropic or natural origin favoring the different species of turtles that can nest in these places.
\end{abstract}


Keywords: marine turtles; nesting; conservation; sustainability; artificial light.

\section{Introducción}

Las tortugas marinas son un grupo de reptiles que se encuentra en todos los océanos del planeta, en especial en aguas tropicales y templadas, con un ciclo de vida complejo; son organismos sumamente carismáticos y especies bandera para la conservación de los hábitats marino-costeros, también presentan una gran importancia en cuanto a prestación de servicios eco sistémicos se refiere (Comisión Permanente del Pacífico Sur, 2018).

Son múltiples los roles que cumplen en el ecosistema, su alimentación permite trasladar grandes cantidades de carbono a las zonas abisales, lo que contribuye al sumidero de carbono en los océanos; por otro lado, participan en la modificación de hábitats, forman claros en la vegetación marina siendo estos básicos para la dinámica de las sucesiones de especies y presencia de organismos clave en las cadenas tróficas (Rondón, Buitrago y Maccoy, 2009); éstos y otros factores ecológicos hacen que la comunidad científica aporte con esfuerzos para la conservación de estos animales. En la actualidad la Unión Internacional para la Conservación de la Naturaleza (UICN), a través de su Lista Roja de especies amenazadas, ha determinado que seis de las siete especies de tortugas se encuentran con algún grado de amenaza: Tortugas Laúd (Dermochelys coriacea), Boba (Caretta caretta) y Golfina (Lepidochelys olivacea) se ubican como Vulnerables; la Tortuga Verde (Chelonia mydas) ha sido catalogada En Peligro; la Tortuga Carey (Eretmochelys imbricata) y Bastarda (Lepidochelys kempii) han sido ubicadas en la categoría Peligro Crítico (WWF, 2019). En Ecuador se registran cuatro de las siete especies de tortugas marinas existentes a escala mundial: Tortuga Verde, Tortuga Carey, Tortuga Laúd y Tortuga Golfina (Muñoz, 2009).

Es importante mencionar que existe un marco legal, así como políticas de conservación y manejo de estas especies; muestra de ello fue que en 1990 el estado ecuatoriano prohibió la captura, procesamiento y comercialización interna y externa de ejemplares de estas especies (Mizobe y Contreras, 2014). Si bien existen diferentes mecanismos de protección, todavía persisten diferentes amenazas pudiendo ser directas como la pesca accidental, el consumo ilegal de las tortugas y uso del caparazón para distintos fines; así como amenazas indirectas: el desarrollo de infraestructuras, luz artificial, deforestación, contaminación química, contaminación por desechos sólidos, turismo, cambio climático (Sarlo, 2017), lo cual ha alterado la estructura de las playas y la dinámica de las zonas de forrajeo y anidación (Coello y Herrera, 2011; Ruiz, Díaz y Merel, 2007).

Por esta razón la presente investigación busca evaluar una de las actividades antropogénicas que causa problemas en las costas ecuatorianas como es la luz artificial y su posible impacto en la anidación de las tortugas marinas, conociendo que las zonas pobladas cerca del Parque Nacional Machalilla, Puerto López y Salango, en los últimos años han generado una gran demanda turística, reconociendo que el turismo más allá de ser una actividad de entretenimiento es el sustento de miles de personas y promueve el desarrollo económico, social y cultural de una localidad (Piedra y Mora, 2005). 
Resaltamos la necesidad de integrar y socializar la conciencia e importancia del medio ambiente, en localidades donde la principal actividad es la visita y aprovechamiento de los recursos naturales, pudiendo identificar la poca iniciativa colaborativa de gobiernos locales y administrativos de las zonas protegidas. En el cantón Puerto López se ha constatado un incremento en actividades de recreación nocturna y esparcimiento social sin un adecuado control y respeto a la vida silvestre, este punto es muy importante ya que investigaciones de zonas muy parecidas como Playas en Costa Rica han demostrado que en áreas de anidamiento donde existe luz artificial se ve afectado el comportamiento de las hembras, así como la eclosión de neonatos, ya que Sánchez (2007), Piedra y Morales (2015) y Rondón et al. (2009) mencionan que la anidación de las tortugas se relaciona con determinado ciclo lunar, la eclosión de las crías se presenta en la fase de cuarto creciente o luna llena, permitiendo que los neonatos encuentren la dirección al mar siguiendo el resplandor de la luz de la luna reflejada en las olas del mar, es por eso que la luz artificial pudiera desorientar a las tortugas recién salidas de los huevos que buscan la dirección del mar y al seguir erróneamente la luz artificial se exponen a depredadores o a la deshidratación por no llegar en un tiempo adecuado al agua (Witherington y Martín, 2003); por otro lado, la luz artificial confunde a las tortugas hembras que se encuentren en el mar reduciendo la cantidad de hembras que finalmente anidan (Piedra y Morales, 2015); por lo tanto, el presente estudio es un acercamiento para describir la contaminación lumínica en las costas ecuatorianas y su efecto sobre las tortugas marinas.

\section{Materiales y Métodos}

\section{Área de estudio}

La investigación se llevó a cabo en la Provincia de Manabí en la costa central del Ecuador, en el Cantón Puerto López, ubicado entre $01^{\circ} 10^{\prime}$ y $01^{\circ} 40^{\prime}$ de latitud sur y entre los $80^{\circ} 25^{\prime}$ y $80^{\circ} 52^{\prime}$ de longitud occidental (Figura 1). Siendo seleccionadas cuatro playas: Puerto López, Playita, Salango y Piqueros, de las cuales Playita forma parte del Parque Nacional Machalilla; el cantón Puerto López está conformado por la cabecera cantonal Puerto López y las cabeceras parroquiales Machalilla y Salango, perteneciendo el 60 \% del cantón al territorio del Parque Nacional Machalilla (GAD Municipal del Cantón Puerto López, 2014). 


\section{Figura 1}

\section{Ubicación de las playas muestreadas y los poblados aledaños}

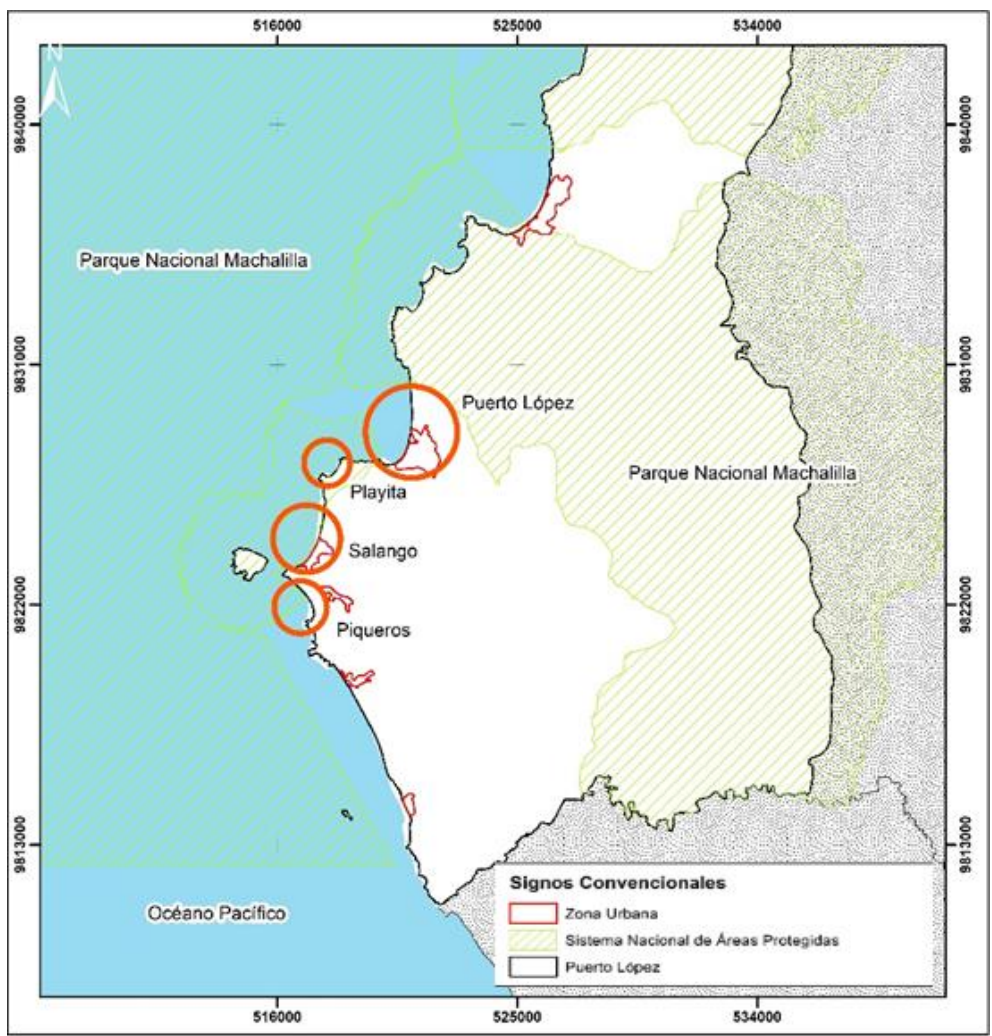

Con relación a las principales actividades socioeconómicas, la mayor ocupación de la población es el turismo, seguido por la pesca que representa el rubro más importante en la economía local del cantón. La superficie cantonal destinada a las actividades agropecuarias representa apenas el 3,45 \% de la superficie total del cantón. La actividad comercial se concentra en la cabecera cantonal Puerto López y en algunos poblados del cantón como Machalilla y Salango (GAD Municipal del Cantón Puerto López, 2014).

El objetivo principal del estudio fue determinar los niveles de luz artificial y las consecuencias que podrían tener sobre la anidación de las tortugas marinas en las cuatro playas seleccionadas en el cantón Puerto López.

\section{Metodología}

El procedimiento empleado en cada playa fue el registro de niveles de luz con un luxómetro (SEW lightmeter 2330 LX) cada 50 m, a lo largo de la línea de pleamar y se realizó el registro de los puntos con GPS (Garmin GPSMAP 64st) para posteriormente graficar los resultados; los recorridos se iniciaron desde el lado sur de la playa siguiendo la línea de pleamar 
hasta la zona norte, llevándose a cabo la toma de datos entre las $20 \mathrm{~h} 00$ y $22 \mathrm{~h} 00$ en cada una de las playas, empezando el estudio el 21 de mayo al 7 de junio de 2019, teniendo dos noches de muestreo por playa las cuales se alternaba con las actividades de la Fundación Equilibrio Azul (EA), captura y recaptura de tortugas, monitoreo de playas por posible eclosión de tortugas y registro de captura incidental de tiburones.

De manera paralela, se obtuvieron los datos del porcentaje de anidación en los distintos sectores de cada playa facilitados por la Fundación Equilibrio Azul (EA) que lleva un registro y monitoreo de las tortugas marinas en áreas del Parque Nacional Machalilla. Para determinar si existe una relación de dependencia entre los datos realizamos una correlación lineal de Pearson.

\section{Resultados}

\section{Playa de Puerto López}

En esta playa se encuentra una zona con una barrera natural contra la luz artificial, lo cual provoca que esta se prolongue con la presencia de zonas sin contaminación lumínica.

\section{Tabla 1}

Incidencia de la luz artificial en áreas de anidación de tortugas en la Playa de Puerto López

\begin{tabular}{clcc}
\hline \multicolumn{4}{c}{ Puerto López } \\
\hline Zona & \multicolumn{1}{c}{ Zona de anidación } & Porcentaje de nidos & Promedio de lúmenes \\
\hline PL7 a PL8 & La Poza & $36,35 \%$ & 0,5575 \\
PL6 a PL7 & Cabañas & $4,55 \%$ & 0,1043 \\
PL5 a PL6 & Retén Naval & $4,55 \%$ & 0,2173 \\
PL4 a PL5 & Hostería Mandala & $13,64 \%$ & 0,0344 \\
PL3 a PL4 & Ramada Arena & $9,09 \%$ & 0 \\
PL2 a PL3 & Previo al Centro Rescate PNM & $18,18 \%$ & 0 \\
PL1 a PL2 & Punta Norte & $13,64 \%$ & 0 \\
\hline
\end{tabular}

Nota. Autoría propia

La Corelación de Pearson entre el porcentaje de nidos y promedio de lumenes tiene un coeficiente de corelacion lineal de 0,4127 y un coeficiente de determinacion 0,1703. 


\section{Figura 2}

Gráfica de la corelación de Pearson de la información en la playa de Puerto López.

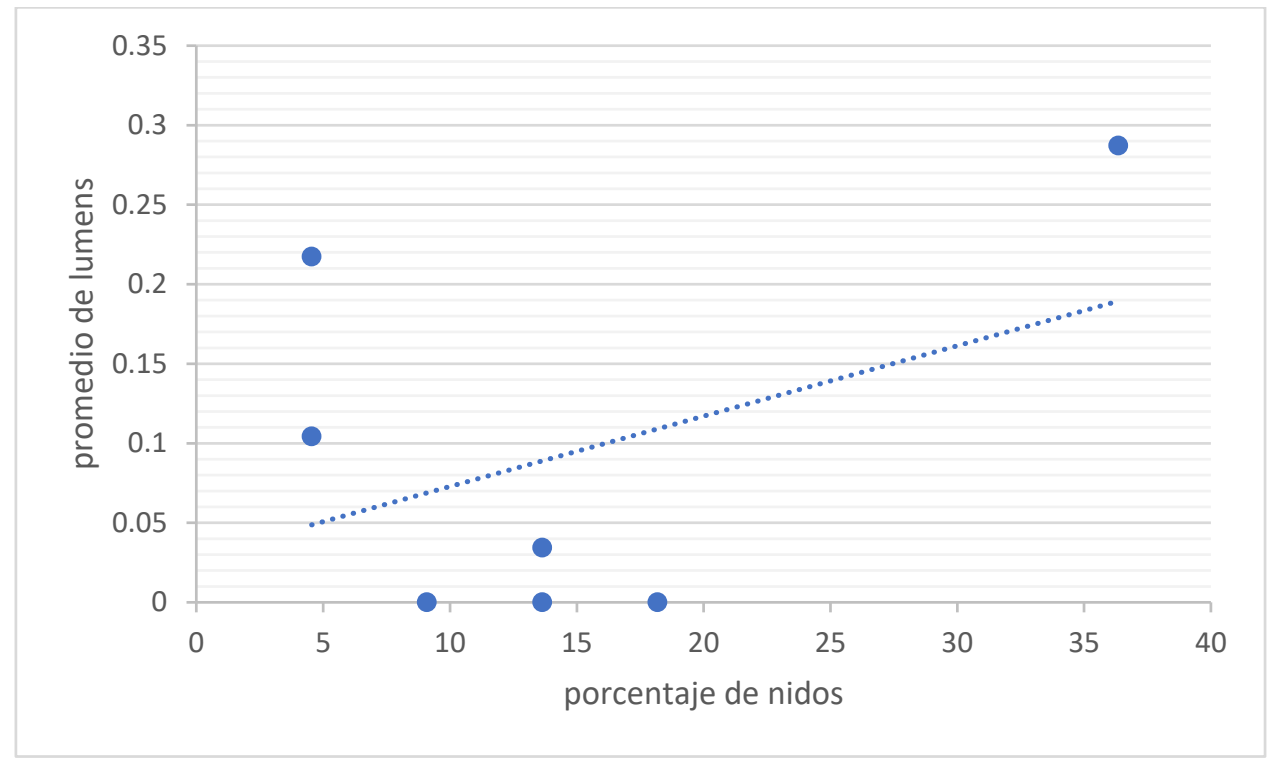

\section{Figura 3}

Distribución de la intensidad lumínica en la Playa de Puerto López y su relación con las áreas de anidación

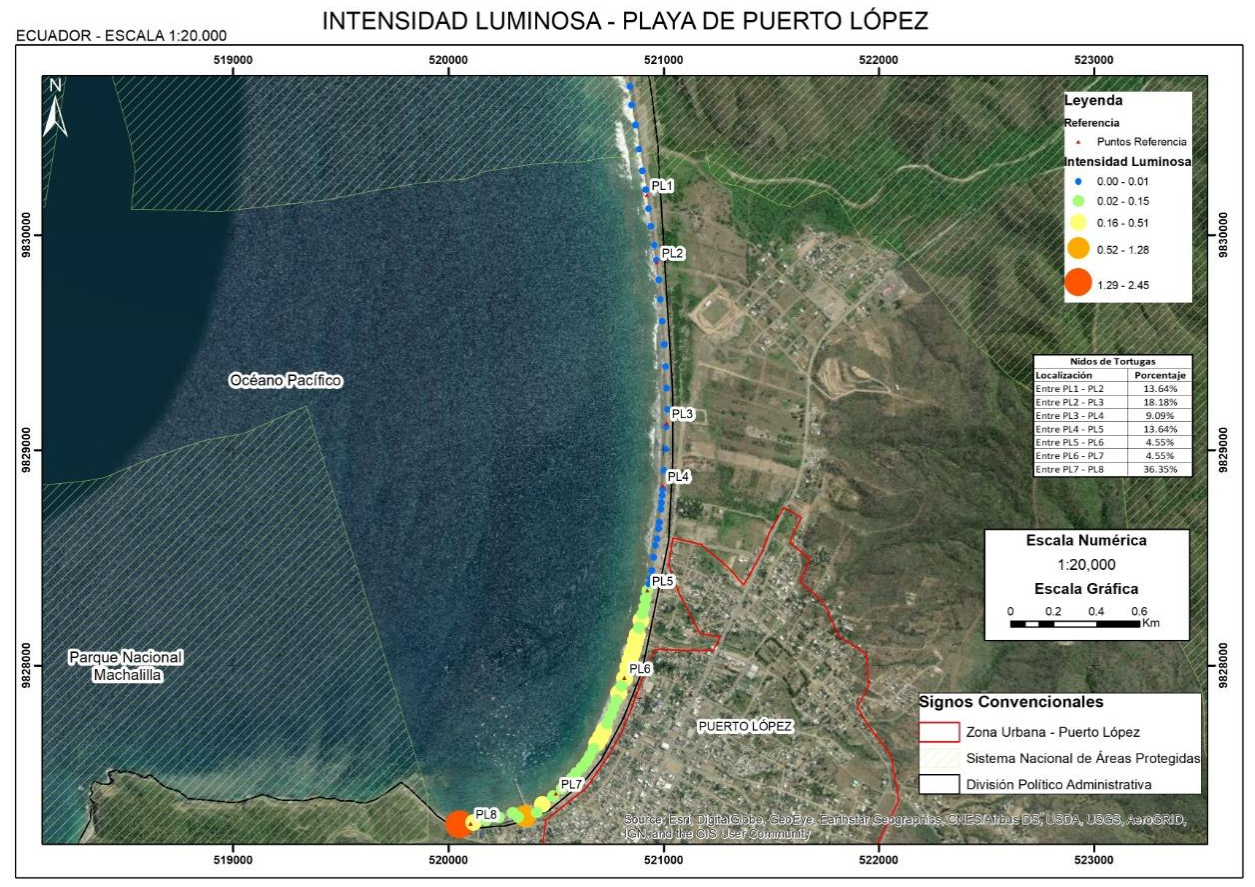




\section{Playa de Salango}

En esta playa se presenta una pequeña zona con una barreara de origen antrópico contra la luz artificial, siendo esta barrera el cementerio local, el cual no suprimió en su totalidad la luz, pero generó una caída en la intensidad de la luz, e incrementó el porcentaje de nidos en la zona, comparado con las zonas que flanquean al cementerio.

\section{Tabla 2}

Incidencia de luz artificial en áreas de anidación de tortugas - Playa Salango

\begin{tabular}{llcc}
\hline \multicolumn{3}{c}{ Salango } \\
\hline \multicolumn{1}{c}{ Zona } & \multicolumn{1}{c}{ Zona de anidación } & Porcentaje de nidos & Promedio de lúmenes \\
\hline SA10 a SA11 & Entre fabrica y punta sur & $4,35 \%$ & 0,3592 \\
SA9 a SA10 & Zona poblada & $0 \%$ & 1,1208 \\
& Entre 3er camino & $13,04 \%$ & 0,0692 \\
SA8 a SA9 & cementerio & & 0,0086 \\
SA7 a SA8 & Entre 2do_3er camino & $8,7 \%$ & 0 \\
SA6 a SA7 & Entre 1er_2do camino & $8,7 \%$ & 0 \\
SA5 a SA6 & 1er camino & $8,7 \%$ & 0 \\
& Zona norte antes del & $43,48 \%$ & 0 \\
SA4 a SA5 & acantilado & $4,35 \%$ & 0 \\
SA3 a SA4 & 2da playa sur & $4,35 \%$ & 0 \\
SA2 a SA3 & 2da playa centro & $4,35 \%$ & \\
SA1 a SA2 & 2da playa norte & & \\
\hline
\end{tabular}

Nota. Autoría propia

La Corelación de Pearson entre el porcentaje de nodos y promedio de lúmenes tiene un coeficiente de corelacion lineal de -0,3297 y un coeficiente de determinacion 0,1087.

\section{Figura 4}

Gráfica de la corelación de Pearson de la información en la playa de Salango

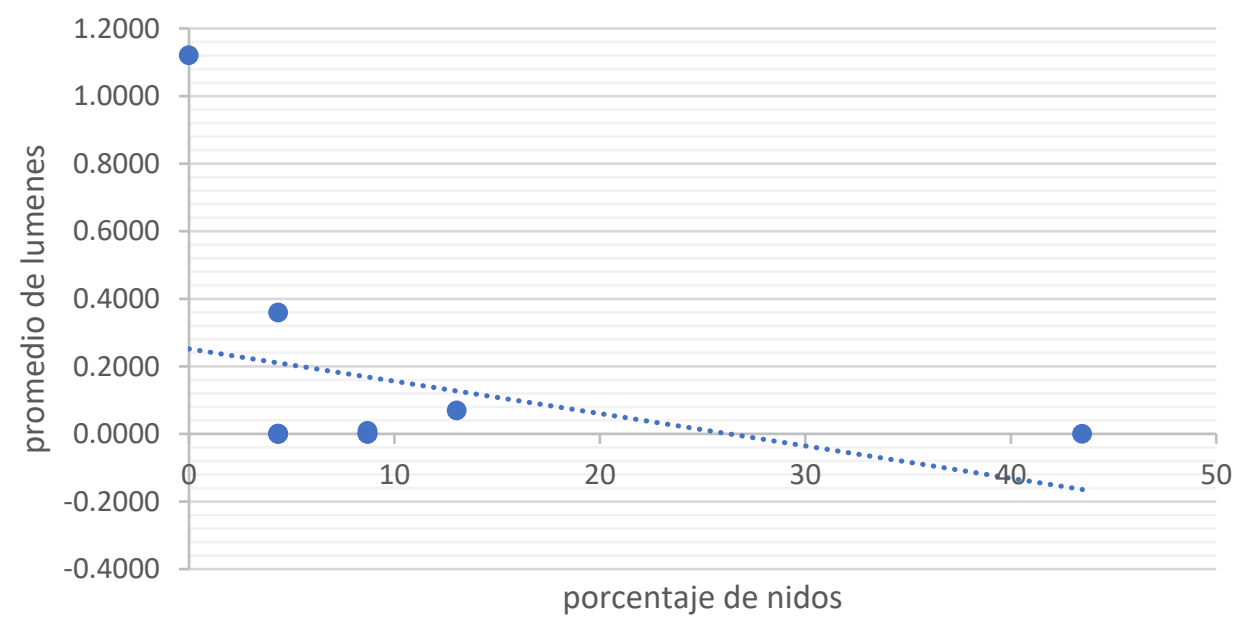

Esta obra se comparte bajo la licencia Creative Common Atribución-No Comercial 4.0 International (CC BY-NC 4.0) Revista de la Universidad Internacional del Ecuador. URL: https://www.uide.edu.ec/ 


\section{Figura 5}

Intensidad lumínica en Playa Salango y su relación con los sitios de nidificación de las tortugas marinas.

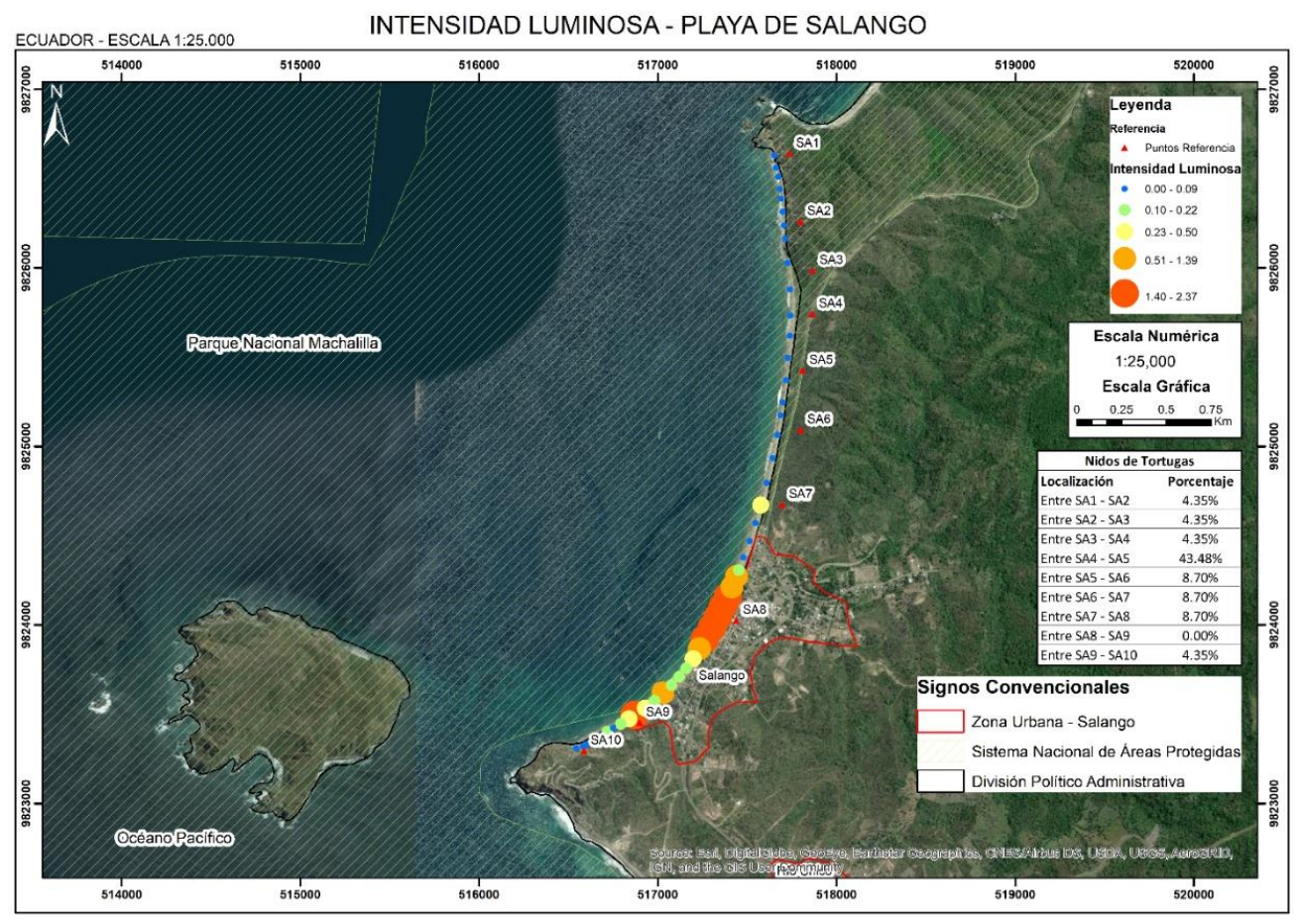

\section{Playa - La Playita}

Se puede apreciar que en esta playa no existe contaminación lumínica por lo cual los datos de los nidos son de origen aleatorio o ligado a parámetros que no fueron abordados en este estudio.

\section{Tabla 3}

Incidencia de la luz artificial en áreas de anidación de tortugas - La Playita

\begin{tabular}{llcc}
\hline \multicolumn{1}{c}{ Zona } & Zona de anidación & Porcentaje de nidos & Promedio de lúmenes \\
\hline PY11 a PY12 & $1 \_2$ & $1,99 \%$ & 0 \\
PY10 a PY11 & $2 \_3$ & $9,77 \%$ & 0 \\
PY9 a PY10 & $3 \_4$ & $12,11 \%$ & 0 \\
PY8 a PY9 & $4 \_5$ & $7,15 \%$ & 0 \\
PY7a PY8 & $5 \_6$ & $6,58 \%$ & 0 \\
PY6 a PY7 & $6 \_7$ & $4,14 \%$ & 0 \\
PY5 a PY6 & $7 \_9$ & $8,22 \%$ & 0 \\
PY4 a PY5 & $8 \_9$ & $9,34 \%$ & 0 \\
PY3 a PY4 & $9 \_10$ & $6,9 \%$ & 0 \\
PY2 a PY3 & $10 \_11$ & $3,22 \%$ & 0 \\
PY1 a PY2 & $11 \_12$ & $30,6 \%$ & 0 \\
\hline
\end{tabular}

Nota. Autoría propia 
La Corelación de Pearson entre el porcentaje de nidos y promedio de lumenes no se aplica en esta playa por no existir la presencia de luz; es decir no se puede establecer ningún sentido de covariación.

\section{Figura 6}

Intensidad lumínica en Playita y su relación con los sitios de nidificación de las tortugas marinas.

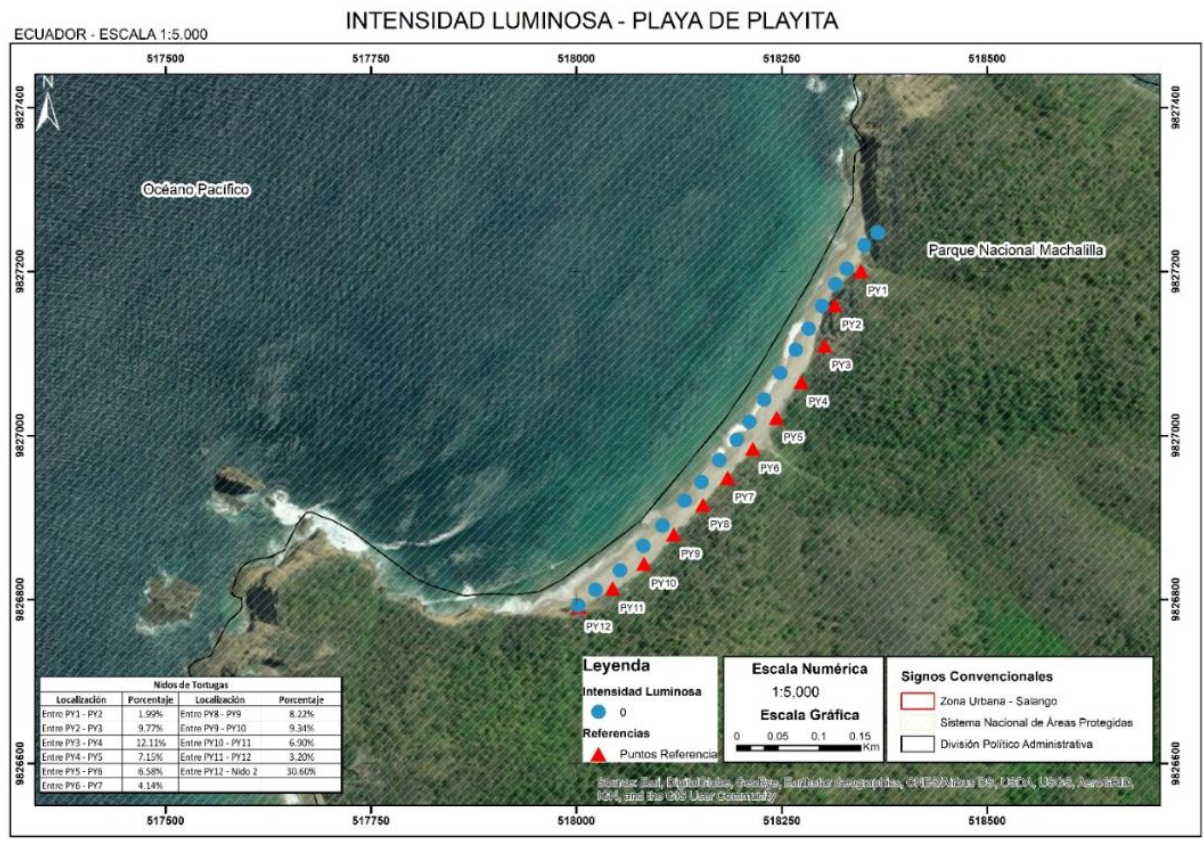

\section{Playa Piqueros}

Se puede apreciar que, en esta playa, al igual que en la Playita, no existe contaminación lumínica por lo cual los datos de los nidos son de origen aleatorio o ligado a parámetros que no fueron abordados en este estudio.

\section{Tabla 4}

Incidencia de la luz artificial en áreas de anidación de tortugas - Playa Piqueros

\begin{tabular}{clcc}
\hline Zona & \multicolumn{1}{c}{ Zona de anidación } & Porcentaje de nidos & Promedio de lúmenes \\
\hline Pq6 a Pq7 & Punta sur & $16,67 \%$ & 0 \\
Pq5 a Pq6 & Playa Amador & $33,33 \%$ & 0 \\
Pq4 a Pq5 & Bocana manglar & $8,33 \%$ & 0 \\
Pq3 a Pq4 & Playa patrick & $16,67 \%$ & 0 \\
Pq2 a Pq3 & 1er acantilado & $16,67 \%$ & 0 \\
Pq1 a Pq2 & Punta norte & $8,33 \%$ & 0 \\
\hline
\end{tabular}

Nota. Autoría propia 
La Corelación de Pearson entre el porcentaje de nidos y promedio de lúmenes no se aplica en esta playa por no existir la presencia de luz; es decir no se puede establecer ningún sentido de covariación.

\section{Figura 7}

Intensidad lumínica en la playa Piqueros y su relación con los sitios de nidificación de las tortugas marinas

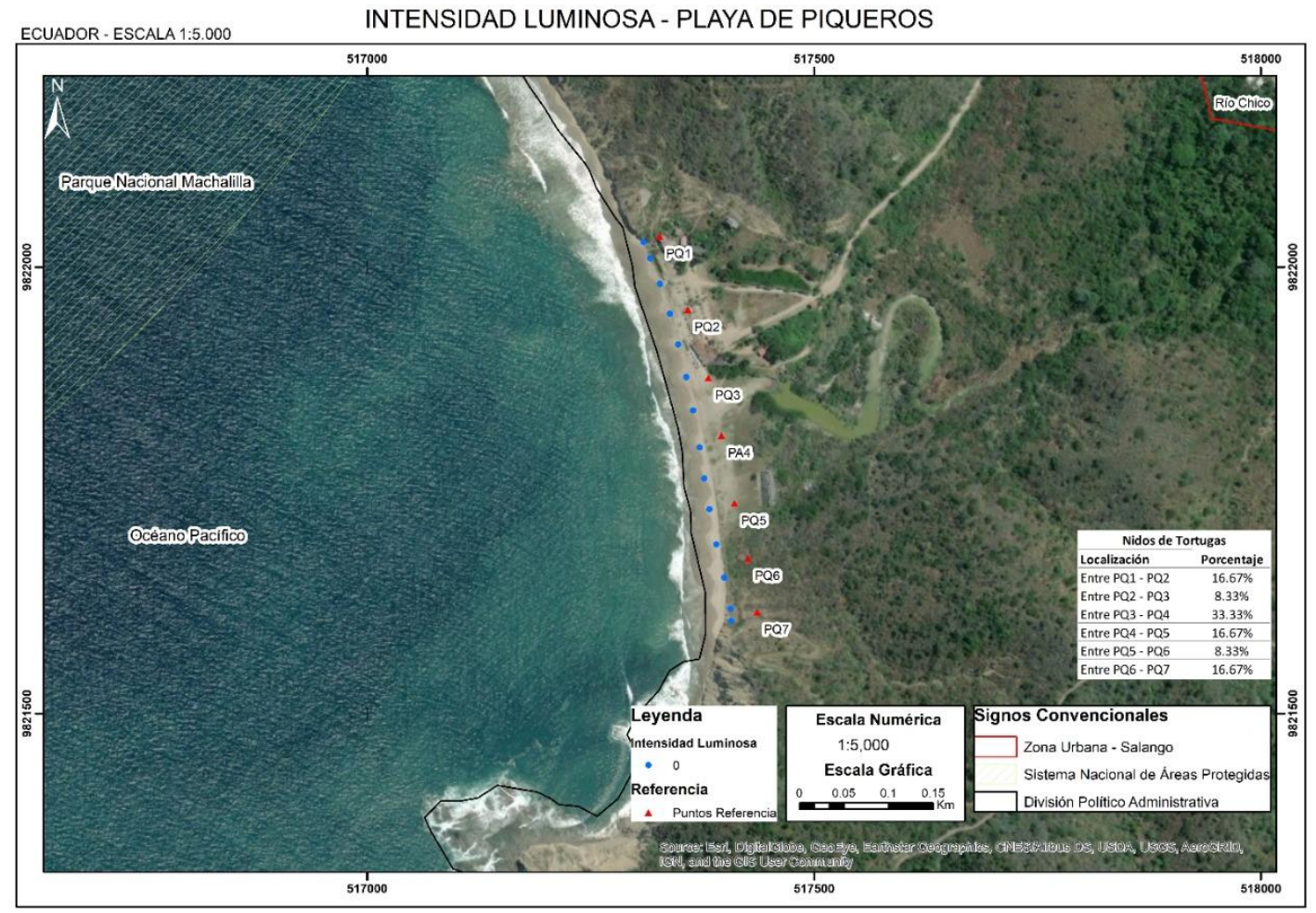

\section{Discusión y Conclusiones}

\section{La luz artificial en áreas de anidación de tortugas - Playa de Puerto López}

Desde el punto de vista del impacto del desarrollo urbanístico, la playa en la localidad Puerto López es la de mayor afectación entre las incluidas en el estudio, la presencia de locales de servicio de bebidas y diversión nocturna en la arena, sumado a la reciente construcción del malecón, conlleva a la presencia de luminarias artificiales relativamente abundantes en la playa.

En las zonas de anidación PL8-PL7-PL6 se obtuvieron los mayores registros de intensidad lumínica, estos valores se pueden asociar a la presencia de postes de luz localizados a una menor distancia de la zona de pleamar; por otro lado, en la zona PL8 en su parte sur es área rocosa y se extiende hasta el muelle turístico, fue la zona con más registros de nidos de tortuga con un 36,35 \% de nidos; en la siguiente zona que se extiende desde PL8 hasta PL7, que comprende el lado norte del muelle turístico, pasando por el mercado de pescado en el que se 
encuentran las lanchas de pescadores, seguido de las covachas de venta de bebidas y entretenimiento nocturno, se registró un rango medio de luz con niveles de 0,02-0,51 lux, presentó un porcentaje de 4,55 \% de anidación de tortugas; le sigue la zona que comprende desde PL6 hasta PL5, donde paulatinamente se registró un descenso de la influencia lumínica, considerando que esta zona comprende estructuras como el retén naval hasta las últimas infraestructuras hoteleras, tomando en cuenta que los registros muestran una disminución importante del porcentaje de nidos registrados, ubicándose en esta área locales nocturnos como discotecas con altos niveles de ruido e iluminación nocturnos; posteriormente desde el punto PL5 hasta PL1 los rangos de luz artificial están en el rango de 0 a 0,1 lux, en esta área el urbanismo se encuentra retirado del área de la playa, además de existir una barrera de vegetación que impide el paso de la luz de las construcciones contiguas al área de la playa (Tabla 1). Por otro lado, existió un porcentaje importante de nidos registrados entre PL3 - PL2 con 18,18 \%, y de PL2 - PL1 con $13,64 \%$, respectivamente.

El coeficiente de Pearson indica una correlación positiva moderada, debido a que el promedio de lúmenes de la zona de la Poza genera ruido en la interpretación de los resultados, debido a que el resultado (en esta zona) es directamente proporcional y eso no es lo que se ha observado en este estudio. La razón es que los datos de anidación de tortugas son históricos y los lúmenes registrados son datos actuales y corresponden a la reciente creación del malecón y muelle pesquero. El coeficiente de correlación lineal nos presenta un valor de 0,1703 mostrando una correlación positiva muy baja, a su vez presenta un coeficiente de determinación de 0,1703 que significa un 17,03 \% de ajuste de las variables; indicando que existe una baja relación de las variables y que el efecto de la luz artificial sobre la anidación no es muy fuerte.

\section{La luz artificial en áreas de anidación de tortugas - Playa Salango}

En Salango, si bien la población no supera los 3350 habitantes existe la tendencia a que el desarrollo urbanístico se encuentre asociado con el área de la playa, sobre todo porque esta población en su mayoría se dedica al arte de la pesca, buceo artesanal y, en menor proporción, al servicio turístico.

En el área de mayor concentración del desarrollo urbanístico, el registro de intensidad lumínica es mayor, esto se refleja en entre las zonas SA10-SA9, las cuales registran los valores más altos de la playa, los mismos que demuestran con datos la presencia de zonas de anidación con los siguientes porcentajes, SA11 - SA10 el 4,35 \%, zona comprendida desde la punta sur hasta la fábrica de harina de pecado (Empresa Pesquera Polar S.A.), seguida de la zona de SA10 - SA9, comprendida toda la zona urbana hasta el cementerio de Salango con luminarias cercanas al área de pleamar, donde no se registran nidos, por otro lado desde SA9 - SA8, se registra un total de $13,04 \%$ de nidos de tortugas, lo que significa que potencialmente esta área de nidificación está siendo afectada por la concentración de los postes de electricidad, lo que probablemente influirá en la disminución del porcentaje de nidos en los próximos años en esta área. A partir de los puntos SA8 a SA2 el registro de intensidad de luz abarca de (0 a 0,09 lux) y se aprecia en el mapa que en esta área no se evidencian construcciones aglomeradas, todo lo contrario, hay un parche de vegetación que protege la playa. 
Cuando se revisa el registro de nidos para estos puntos, se encuentra que el mayor porcentaje de nidos se reporta para la zona comprendida entre SA6 - SA5 con el 43,48 \%, y de SA5 - SA2 con el 4,35 \% de presencia de nidos.

Es importante mencionar que el monitoreo de nidos es realizado por voluntarios por lo que la variación encontrada entre los tramos puede deberse a inconsistencia en el registro. Sin embargo, es evidente que la concentración de la intensidad de luz artificial en la playa está ubicada donde se concentra el urbanismo y que los sitios de nidificación serán desplazados de continuar este desarrollo sin ningún tipo de protección hacia las especies vulnerables como las tortugas marinas.

El coeficiente de Pearson indica una correlación negativa baja; que indica una correlación inversamente proporcional de los datos (a menor luz mayor cantidad de nidos de tortugas). Esta playa no ha sufrido cambios resientes proporcionando una estabilidad temporal en la intensidad de luz. El coeficiente de correlación lineal presente de -0,3297 nos entrega una correlación negativa baja, a su vez presenta un coeficiente de determinación de 0,1087 también interpretado como $10,87 \%$ mostrando un porcentaje bajo de ajuste de las variables; indicando que existe una baja relación de las variables y que el efecto de la luz artificial sobre la anidación no es muy fuerte.

\section{La luz artificial en áreas de anidación de tortugas - La Playita}

La Playita es una pequeña playa perteneciente al Parque Nacional Machalilla, en la que se tiene que recorrer un sendero de 1,4 $\mathrm{km}$ y un total de 283 gradas en zona de bosque seco tropical para acceder a la playa, la cual tiene una extensión aproximada de $1 \mathrm{~km}$ de longitud, allí se realizaron los registros de intensidad lumínica, y se tiene reportes de anidación de tortugas marinas y especial de la tortuga Carey (Eretmochelys imbricata), por lo mismo en la actualidad se encuentra cerrada al público.

Los registros que se obtuvieron en la Playita, demuestran que es una playa en estado natural, que no posee impacto lumínico artificial, ya que se encuentra alejada de la carretera principal, mostrando 0,0 lux en toda la playa, se dividió en zonas de sur a norte, que comprenden entre los puntos PY1 - PY12, el mayor porcentaje de nidos estuvo en la zona entre PY11 PY12 con el 30,6 \%, y el menor en la zona PY1 - PY2 con 1,99 \%, esta es la playa mejor monitoreada por parte del Ministerio del Ambiente y por organizaciones sin fines de lucro asociadas, la variación en el porcentaje de anidación puede deberse a factores no analizados en esta investigación como la influencia de las corrientes marinas o factores físicos de la playa.

Al no existir valores de luz en esta playa no existe una relación lineal entre las variables; pero este resultado es ideal porque uno de los parámetros que desorientan a los neonatos en su camino del nido al océano es la presencia de luz artificial; por lo tanto, el valor cero de lúmenes es determinante para buenos porcentajes de anidación.

\section{La luz artificial en áreas de anidación de tortugas - Playa Piqueros}


La Playa Piqueros, es una playa privada que se encuentra en la localidad de Río Chico a 10,1 km al sur de Puerto López, allí se apreció en las visitas de campo que no existe contaminación lumínica artificial, ya que al momento la infraestructura que antes era un hotel se encontraba en remodelación, y no existía actividad turística, la parte muestreada corresponde a los $500 \mathrm{~m}$ que en marea alta quedan descubiertos y se puede acceder con facilidad.

Se revisaron las zonas desde los puntos PQ1 - PQ7 donde se registró 0,0 lux de luz artificial, teniendo registros de anidación de tortugas marinas en la zona: el área con más alto porcentaje de anidación se encontró entre los puntos PQ5 - PQ6: 33,33 \% y el menor en las zonas comprendidas entre PQ4 - PQ5 y PQ1 - PQ2 que registraron cada uno 8,33 \% de nidos de tortugas.

Al no existir valores de luz en esta playa no existe una relación lineal entre las variables; pero este resultado es ideal porque uno de los parámetros que desorientan a los neonatos en su camino del nido al océano es la presencia de luz artificial; por lo tanto, el valor cero de lúmenes es determinante para buenos porcentajes de anidación.

Conforme con los datos de anidación facilitados por la Fundación Equilibrio Azul (EA) y el registro de luz generado en el trabajo de campo, se pudo constatar que las zonas con menos influencia de luz artificial presentan mayor cantidad de anidación de tortugas marinas, varios estudios como los de Alfaro et al. (2011) y Witherington y Martín (2003) presentan similitudes con los resultados de esta investigación; por otro lado, Blanco y Santidrián (2011) menciona los efectos de la luz artificial en la distribución de los nidos al provocar que las tortugas decidan no salir a poner los huevos por la presencia de contaminación lumínica.

Existen otros factores antropogénicos que se deben considerar para futuras investigaciones como los efectos de la compactación de la arena en la playa por el tráfico de turistas, lo cual dificulta la salida de los neonatos de sus nidos; otra consecuencia es el efecto que causan las infraestructuras antrópicas para actividades turísticas y pesqueras en sitios claves como zonas de descanso y forrajeo para las especies de tortugas registradas en Puerto López (Obs. Cristina Miranda EA 2019), además, en el presente estudio se registró que en la playa de Puerto López existe una barrera de palmeras sembradas en la zona norte al final del malecón (P15), entre el borde de la playa y las construcciones, que crea una sombra en dirección al mar, esto se evidencia con la medición de luz que tuvo como resultado 0,0344 lux con un 13,64\% de nidos, porcentaje similar al zona más alejada del pueblo (Punta Norte), y en el caso de la playa de Salango al tener una barrera artificial como el cementerio $(\mathrm{Sa} 7)$ realiza el mismo efecto de protección, desembocando en un 13,02 \% de nidos; en contraste las otras dos playas muestreadas Piqueros y La Playita, las cuales no tienen influencia de luz artificial y presentan una barrera natural de vegetación silvestre con una distribución más homogénea, esto nos hace confirmar las observaciones de Francia (2004), quien registra que en Costa Rica se observa que las hembras de tortugas prefieren anidar en la sombra proyectada por objetos naturales o artificiales como palmeras y edificios en construcción. 


\section{Referencias Bibliográficas}

Alfaro, M., Zeledón, R., Mora, E., Bolaños, R., y Pucci, J. J. (2011). Tortugas marinas en Costa Rica. Ambientales, 41, 3-4.

Blanco, G., y Santidrián, P. (2011). La tortuga marina negra de Guanacaste amenazada de extinción por prácticas humanas. Tortugas marinas en Costa Rica, Ambientales, 41, 19-26.

Coello, D., y Herrera, M. (2011). Línea Base de Conocimiento sobre el Estado Actual de las Tortugas Marinas en el Ecuador. Instituto Nacional de Pesca, Boletín Especial, 2(2). Recuperado https://www.oceandocs.org/bitstream/handle/1834/4565/Informe\%20de\%20consultoria\% 205\%20de\%20septi\%20publi.pdf?sequence=

Comisión Permanente del Pacífico Sur. (2018). Protección de Tortugas Marinas. Recuperado de: http://cpps-int.org/index.php/2014-09-05-20-06-33/tortugas-marinas

GAD Municipal del Cantón Puerto López. (2014). Plan de Desarrollo y Ordenamiento Territorial. 302pp. Recuperado de http://app.sni.gob.ec/snilink/sni/PORTAL_SNI/data_sigad_plus/sigadplusdocumentofinal/1360003300001_PDY OT_PUERTO_LOPEZ_2014_2019_14-04-2015_20-35-13.pdf

Francia, A. G. (2004). Incidencia humana sobre la anidación de tortugas marinas: recolección de huevos y desarrollo urbano en playa Junquillal, Guanacaste, Costa Rica. Programa regional en manejo de vida silvestre para Mesoamérica y el Caribe (Tesis de pregrado, sin publicar). Universidad Nacional, Heredia, Costa Rica.

Mizobe, C., y Contreras, M. (2014). Anidación de tortugas marinas en la Provincia de Manabí, Ecuador. Revista La Técnica, 12, 38-55. doi:10.33936/la_tecnica.v0i12.592

Muñoz, J. (2009). Identificación y estudio preliminar de los sitios críticos para anidación, forrajeo y descanso de las tortugas marinas en la costa centro y norte del Ecuador (Tesis de pregrado). Universidad San Francisco de Quito, Quito, Ecuador. Recuperado de http://repositorio.usfq.edu.ec/bitstream/23000/1116/1/90849.pdf

Piedra, E., y Mora, N. (2005). Análisis del sistema turístico del cantón Puerto López y determinación de una propuesta para la optimización del funcionamiento de dicho sistema (Tesis de pregrado). Escuela Politécnica del Litoral, Guayaquil, Ecuador. Recuperado http://www.dspace.espol.edu.ec/bitstream/123456789/4558/1/7079.pdf

Piedra, L., y Morales, V. (2015). Preferencias en la anidación de tortugas carey (Eretmochelys imbricata) y baulas (Dermochelys coriacea) en el Refugio Nacional de Vida Silvestre Gandoca Manzanillo, Limón, Costa Rica. Tecnología en Marcha, 28(3), 86-98.

Rondón, M., Buitrago, J., y Mccoy, M. (2009). Impacto de la luz artificial sobre la anidación de la tortuga marina Dermochelys coriacea (Testudines: Dermochelyidae), en playa Cipara, Venezuela. Revista de Biología Tropical, 57(3), 515-528. Recuperado de https://www.scielo.sa.cr/scielo.php?script=sci_arttext\&pid=S0034-77442009000300006

Ruiz, A., Díaz, M., y Merel, R. (2007). WIDECAST Plan de Acción para la Recuperación de las Tortugas Marinas del Caribe de Panamá (Informe Técnico del PAC No. 47). Recuperado de https://www.widecast.org/Resources/Docs/STRAP_Panama_2007.pdf

Sarlo, M. (2017). Diagnóstico de la Situación de las Tortugas Marinas en Panamá y Plan de Acción Nacional para su Conservación. Ministerio de Ambiente de Panamá Conservación Internacional. Recuperado de http://marviva.net/sites/default/files/documentos/diagnostico_situacion_tortugas_marinas _panama_y_pan_conservacion_17-04.pdf 
Witherington, B., y Martín, R. (2003). Entendiendo, evaluando y solucionando los problemas de contaminación de luz en Playas de Anidamiento de Tortugas Marinas. Reporte Técnico. Florida Fish and Wildlife Conservation Commission.

WWF. (2019). Por un futuro en el que los humanos vivan en armonía con la naturaleza. Recuperado de https://www.wwf.es/nuestro_trabajo_/especies_y_habitats/tortugas_marinas/ 